\title{
Oxidative Rearrangement of Cyclic Tertiary Allylic Alcohols with IBX in DMSO
}

\author{
Masatoshi Shibuya, Shinichiro Ito, Michiyasu Takahashi and Yoshiharu Iwabuchi* \\ Graduate School of Pharmaceutical Sciences, Tohoku University \\ Aobaayama, Sendai 980-8578, Japan \\ Fax: $+81-22-217-6845$ \\ E-mail: iwabuchi@mail.pharm.tohoku.ac.jp
}

\section{Supporting Information}

\section{Table of Contents}

I. General

$\mathrm{S} 2$

II. Synthesis of tertiary allylic alcohol substrates

III. Typical experimental procedure for IBX mediated oxidative rearrangement S8-S11

IV. References

S12

V. NMR spectra

S13-65

\section{General.}

IBX, ${ }^{1}$ Dess-Martin periodinane, ${ }^{2} \mathrm{PhIO}_{2},{ }^{3}$ and $\mathrm{PhIO}^{4}$ were prepared according to the literature procedures. Other chemicals and solvents were either purchased from commercial suppliers and used without purification, except for DMSO distilled over $\mathrm{CaH}_{2}$. All reactions were stirred magnetically, under an argon atmosphere, unless otherwise noted, and monitored with analytical TLC (Merck Kieselgel $60 \mathrm{~F}_{254}$ ). Column chromatography was carried out with silica gel 60 particle size $0.063-0.210 \mathrm{~mm}$. Melting points were taken with Yazawa BY-2 and are uncorrected. NMR spectra were measured in JEOL JNM-AL400 (400 MHz). Chemical 
shifts were reported in the $\delta$ scale relative to tetramethylsilane (TMS) as $0.00 \mathrm{ppm}$ for ${ }^{1} \mathrm{H}$ $\left(\mathrm{CDCl}_{3}\right)$ and residual $\mathrm{CHCl}_{3}\left(7.26 \mathrm{ppm}\right.$ for ${ }^{1} \mathrm{H}$ and $77.00 \mathrm{ppm}$ for ${ }^{13} \mathrm{C}$ ), as internal reference. The infarared (IR) spectra were recorded on JASCO IR-700.

\section{Synthesis of the tertiary allyic alcohol substrates}

Scheme S1

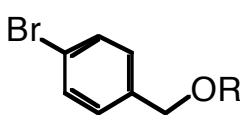

S4: $\mathrm{R}=\mathrm{MOM}$

S6: $R=$ TBDMS

$$
\underset{\text { then 2-cyclohexen-1-one }}{\stackrel{\text { tert-BuLi, THF, }-78^{\circ} \mathrm{C}}{\longrightarrow}}
$$

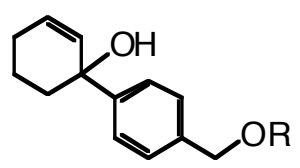

$$
\begin{aligned}
& \text { 4a }: \mathrm{R}=\mathrm{MOM} \\
& \text { TBAF, THF } \quad 6 \mathbf{a}: \mathrm{R}=\mathrm{TBDMS}
\end{aligned}
$$

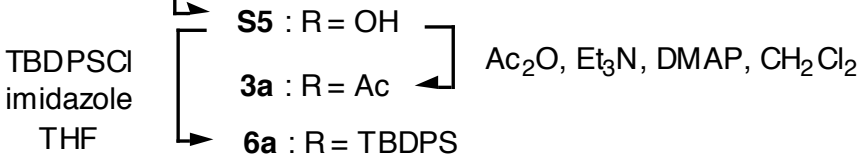

\section{General procedure for 1,2 -addition of organolithium reagents to $\alpha, \beta$-unsaturated} ketones." Phenyllithium (1.0 M in cyclohexane-diethyl ether), butyllithium (1.6 $\mathrm{M}$ in hexane) and methyllithium (1.0M in diethyl ether)were purchased from commercial suppliers. The other aryllithium reagents were prepared in situ from the corresponding aryl bromide by the halogen-lithium exchanging protocol ${ }^{\#}$ using tert-buthyllithium (1.5M in $n$-pentane) in THF at $-78{ }^{\circ} \mathrm{C}$. To a solution of $\alpha, \beta$-unsaturated ketone in THF (concentration $0.2 \mathrm{M}$ ) at $-78{ }^{\circ} \mathrm{C}$ was added organolithium reagent (1.5 equiv). After the mixture was stirred at $-78{ }^{\circ} \mathrm{C}$ for $1 \mathrm{hr}$, $\mathrm{H}_{2} \mathrm{O}$ was added and allowed to warm to $\mathrm{rt}$, and the mixture was extracted with $\mathrm{Et}_{2} \mathrm{O}$. The organic extract was washed with brine, dried $\left(\mathrm{MgSO}_{4}\right)$, and concentrated. The residue was purified by silica gel column chromatography (EtOAc-hexane $=1: 4$ ) to give tert-allylic alcohol.

\section{1-Phenyl-2-cyclohexen-1-ol (1a)}

mp 44-45 ${ }^{\circ} \mathrm{C}$ (recrystallized from hexane); IR (neat): $3398 \mathrm{~cm}^{-1}$; ${ }^{1} \mathrm{H}-\mathrm{NMR} \delta 7.46(\mathrm{~d}, 2 \mathrm{H}, J=7.8 \mathrm{~Hz}), 7.31(\mathrm{t}, 2 \mathrm{H}, J=7.8 \mathrm{~Hz})$, 7.22 (tt, $1 \mathrm{H}, J=7.4,1.2 \mathrm{~Hz}), 6.00(\mathrm{ddd}, 1 \mathrm{H}, J=10.0,3.9,3.7 \mathrm{~Hz})$, $5.75(\mathrm{~d}, 1 \mathrm{H}, J=10.0 \mathrm{~Hz}), 2.16-1.99(\mathrm{~m}, 4 \mathrm{H}), 1.85(\mathrm{dd}, 1 \mathrm{H}, J=$ 12.8, $2.8 \mathrm{~Hz}), 1.82-1.72(\mathrm{~m}, 1 \mathrm{H}), 1.63-1.54(\mathrm{~m}, 1 \mathrm{H}) ;{ }^{13} \mathrm{C}-\mathrm{NMR}$ $\left(100 \mathrm{MHz}, \mathrm{CDCl}_{3}\right) \delta 147.7,132.1,130.4,127.9,126.6,125.3$, 72.1, 39.5, 25.0, 19.2; MS m/z $174\left(\mathrm{M}^{+}\right), 146$ (100\%); HRMS Calcd. $\mathrm{C}_{12} \mathrm{H}_{14} \mathrm{O}:$ 174.1045. Found: 174.1013. 


\section{1-Butyl-2-cyclohexen-1-ol (2a)}

IR (neat): $3377 \mathrm{~cm}^{-1} ;{ }^{1} \mathrm{H}-\mathrm{NMR} \delta 5.78(\mathrm{dt}, 1 \mathrm{H}, J=10.0,4.3 \mathrm{~Hz})$, $5.61(\mathrm{~d}, 1 \mathrm{H}, J=10.0 \mathrm{~Hz}), 1.97(\mathrm{~m}, 2 \mathrm{H}), 1.58(\mathrm{~m}, 7 \mathrm{H}), 1.27(\mathrm{~m}$, $4 \mathrm{H}), 0.91(\mathrm{t}, 3 \mathrm{H}, J=6.3 \mathrm{~Hz}) ;{ }^{13} \mathrm{C}-\mathrm{NMR}\left(100 \mathrm{MHz}, \mathrm{CDCl}_{3}\right) \delta$ 132.8, 129.5, 69.6, 42.0, 35.4, 25.7, 25.2, 23.3, 19.1, 14.1; MS m/z $154\left(\mathrm{M}^{+}\right), 97$ (100\%); HRMS Calcd. $\mathrm{C}_{10} \mathrm{H}_{18} \mathrm{O}: 154.1358$. Found: 154.1342 .

\section{1-(4-Acetoxymethylphenyl)-2-cyclohexen-1-ol (3a)}

IR (neat): 3455, $1739 \mathrm{~cm}^{-1} ;{ }^{1} \mathrm{H}-\mathrm{NMR} \delta 7.48(\mathrm{~d}, 2 \mathrm{H}, J=8.3 \mathrm{~Hz})$,

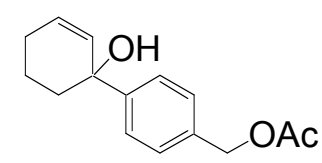

$7.30(\mathrm{~d}, 2 \mathrm{H}, J=8.3 \mathrm{~Hz}), 6.03(\mathrm{dt}, 1 \mathrm{H}, J=10.0,3.2 \mathrm{~Hz}), 5.75(\mathrm{~d}$, $1 \mathrm{H}, J=10.0 \mathrm{~Hz}), 5.09$ (s, 2H), 2.20-1.90 (m, 4H), 2.09 (s, 3H), 1.88-1.72 (m, 2H), 1.70-1.56 (m, $1 \mathrm{H}) ;{ }^{13} \mathrm{C}-\mathrm{NMR}(100 \mathrm{MHz}$, $\left.\mathrm{CDCl}_{3}\right) \delta 170.8,147.9,134.3,131.9,130.8,128.0,125.6,72.0$, 66.1, 39.6, 25.0, 21.1, 19.2; MS m/z $246\left(\mathrm{M}^{+}\right), 186(100 \%)$

\section{1-(4-Methoxymethoxymethylphenyl)-2-cyclohexen-1-ol (4a)}

IR (neat): $3444 \mathrm{~cm}^{-1} ;{ }^{1} \mathrm{H}-\mathrm{NMR} \delta 7.46(\mathrm{dt}, 2 \mathrm{H}, J=8.3 \mathrm{~Hz}), 7.32$ $(\mathrm{d}, 2 \mathrm{H}, J=8.3 \mathrm{~Hz}), 6.02(\mathrm{dt}, 1 \mathrm{H}, J=10.0,3.7 \mathrm{~Hz}), 5.77(\mathrm{~d}, 1 \mathrm{H}, J$ $=10.0 \mathrm{~Hz}), 4.69(\mathrm{~s}, 1 \mathrm{H}), 4.57(\mathrm{~s}, 1 \mathrm{H}), 3.40(\mathrm{~s}, 3 \mathrm{H}), 2.20-2.00(\mathrm{~m}$, $3 \mathrm{H}), 1.96(\mathrm{dd}, 1 \mathrm{H}, J=10.3,8.6 \mathrm{~Hz}), 1.88-1.72(\mathrm{~m}, 2 \mathrm{H}), 1.67-1.58$ $(\mathrm{m}, 1 \mathrm{H}) ;{ }^{13} \mathrm{C}-\mathrm{NMR}\left(100 \mathrm{MHz}, \mathrm{CDCl}_{3}\right) \delta 147.3,136.2,132.0$, 130.6, 127.6, 125.5, 95.5, 72.0, 68.9, 55.3, 39.6, 25.0, 19.2; MS m/z $248\left(\mathrm{M}^{+}\right), 186(100 \%)$; HRMS Calcd. $\mathrm{C}_{15} \mathrm{H}_{20} \mathrm{O}_{3}: 248.1412$. Found: 248.1397. 


\section{1-(4-Hydroxymethylphenyl)-2-cyclohexen-1-ol (S5)}

IR (neat): $3348 \mathrm{~cm}^{-1} ;{ }^{1} \mathrm{H}-\mathrm{NMR} \delta 7.47(\mathrm{dt}, 2 \mathrm{H}, J=8.3,2.07 \mathrm{~Hz}$ ), $7.32(\mathrm{~d}, 2 \mathrm{H}, J=8.3 \mathrm{~Hz}), 6.03(\mathrm{dt}, 1 \mathrm{H}, J=9.8,3.2 \mathrm{~Hz}), 5.76(\mathrm{~d}$, $1 \mathrm{H}, J=9.8 \mathrm{~Hz}), 4.67(\mathrm{~d}, 2 \mathrm{H}, J=3.5 \mathrm{~Hz}), 2.20-1.70(\mathrm{~m}, 6 \mathrm{H}), 1.70-$ $1.55(\mathrm{~m}, 1 \mathrm{H}) ;{ }^{13} \mathrm{C}-\mathrm{NMR}\left(100 \mathrm{MHz}, \mathrm{CDCl}_{3}\right) \delta 147.2,139.3,132.0$, 130.7, 126.7, 125.6, 72.1, 65.1, 39.6, 25.1, 19.2; MS m/z 204 $\left(\mathrm{M}^{+}\right), 145$ (100\%); HRMS Calcd. $\mathrm{C}_{13} \mathrm{H}_{16} \mathrm{O}_{2}$ : 204.1150. Found: 204.1137.

\section{1-[4-(tert-Butyldiphenylsiloxymethyl)-2-cyclohexen-1-ol (5a)}

IR (neat): $3390 \mathrm{~cm}^{-1}$; ${ }^{1} \mathrm{H}-\mathrm{NMR} \delta 7.69(\mathrm{~d}, 4 \mathrm{H}, \mathrm{J}=6.6 \mathrm{~Hz}), 7.44$ $(\mathrm{d}, 2 \mathrm{H}, J=8.1 \mathrm{~Hz}), 7.46-7.32(\mathrm{~m}, 6 \mathrm{H}), 7.32(\mathrm{~d}, 2 \mathrm{H}, J=8.1 \mathrm{~Hz})$, $6.03(\mathrm{dt}, 1 \mathrm{H}, J=10.0,3.7 \mathrm{~Hz}), 5.79$ (d, $1 \mathrm{H}, J=10.0 \mathrm{~Hz}), 2.20-$ $1.95(\mathrm{~m}, 3 \mathrm{H}), 1.90-1.75(\mathrm{~m}, 3 \mathrm{H}), 1.65-1.55(\mathrm{~m}, 1 \mathrm{H}), 1.09(\mathrm{~s}, 9 \mathrm{H})$; ${ }^{13} \mathrm{C}-\mathrm{NMR}\left(100 \mathrm{MHz}, \mathrm{CDCl}_{3}\right) \delta 146.3,139.6,135.5,133.4,132.2$, 130.5, 129.6, 127.6, 125.6, 125.3, 72.1, 65.3, 39.6, 26.9, 25.1, 19.4, 19.3; MS m/z $442\left(\mathrm{M}^{+}\right), 199$ (100\%); HRMS Calcd. $\mathrm{C}_{29} \mathrm{H}_{33} \mathrm{O}_{2} \mathrm{Si}\left(\mathrm{M}^{+}-\mathrm{H}_{2} \mathrm{O}\right)$ : 424.2222. Found: 424.2201.

\section{1-[4-(tert-Butyldimethylsiloxymethyl)phenyl]-2-cyclohexen-1-ol (6a)}

mp 57-58 ${ }^{\circ} \mathrm{C}$ (recrystallized from hexane); IR (neat): $3393 \mathrm{~cm}^{-1}$; ${ }^{1} \mathrm{H}-\mathrm{NMR} \delta 7.44(\mathrm{~d}, 2 \mathrm{H}, J=8.3 \mathrm{~Hz}), 7.28(\mathrm{~d}, 2 \mathrm{H}, J=8.3 \mathrm{~Hz}), 6.01$ $(\mathrm{dt}, 1 \mathrm{H}, J=10.1,3.8 \mathrm{~Hz}), 5.77(\mathrm{~d}, 1 \mathrm{H}, J=10.1 \mathrm{~Hz}), 4.72(\mathrm{~s}, 2 \mathrm{H})$, 2.20-1.88 (m, 3H), $1.92(\mathrm{~s}, 1 \mathrm{H}), 1.88-1.72(\mathrm{~m}, 2 \mathrm{H}), 1.70-1.56(\mathrm{~m}$, $1 \mathrm{H}), 0.93(\mathrm{~s}, 9 \mathrm{H}), 0.09(\mathrm{~s}, 6 \mathrm{H}) ;{ }^{13} \mathrm{C}-\mathrm{NMR}\left(100 \mathrm{MHz}, \mathrm{CDCl}_{3}\right) \delta$ 146.4, 139.9, 132.2, 130.5, 127.6, 125.7, 125.3, 72.1, 64.8, 39.6, 26.0, 25.1, 19.3, -5.1; MS m/z $318\left(\mathrm{M}^{+}\right), 97$ (100\%); HRMS Calcd. $\mathrm{C}_{19} \mathrm{H}_{30} \mathrm{O}_{2} \mathrm{Si}: 318.2015$. Found: 318.1990 . 


\section{1-[3-(tert-Butyldimethylsilanyloxy)phenyl]-2-cyclohexen-1-ol (7a)}

IR (neat): $3398 \mathrm{~cm}{ }^{1} \mathrm{H}-\mathrm{NMR} \delta 7.18(\mathrm{dd}, 1 \mathrm{H}, J=8.0,7.8$ $\mathrm{Hz}), 7.04(\mathrm{~d}, 1 \mathrm{H}, J=7.8 \mathrm{~Hz}), 6.97(\mathrm{~s}, 1 \mathrm{H}), 6.71(\mathrm{~d}, 1 \mathrm{H}, J=8.0$ $\mathrm{Hz}), 6.01(\mathrm{dt}, 1 \mathrm{H}, J=10.0,3.7 \mathrm{~Hz}), 5.75(\mathrm{~d}, 1 \mathrm{H}, J=10.0 \mathrm{~Hz})$, 2.20-2.00 (m, 2H), 1.99-1.89 (m, 2H), 1.89-1.71 (m, 2H), 1.68$1.54(\mathrm{~m}, 1 \mathrm{H}), 0.98(\mathrm{~s}, 9 \mathrm{H}), 0.19(\mathrm{~m}, 6 \mathrm{H}) ;{ }^{13} \mathrm{C}-\mathrm{NMR}(100 \mathrm{MHz}$, $\left.\mathrm{CDCl}_{3}\right) \delta 155.3,149.4,132.0,130.5,128.8,118.3,117.4,72.0$, 39.5, 25.8, 25.0, 19.2, 18.3, -4.3; MS m/z $304\left(\mathrm{M}^{+}\right), 229$ (100\%); HRMS Calcd. $\mathrm{C}_{18} \mathrm{H}_{28} \mathrm{O}_{2} \mathrm{Si}\left(\mathrm{M}^{+}\right)$: 304.1859. Found: 304.1859 .

\section{1-Phenyl-2-cyclopenten-1-ol (8a)}

IR (neat): $3428 \mathrm{~cm}^{-1} ;{ }^{1} \mathrm{H}-\mathrm{NMR} \delta 7.43(\mathrm{~d}, 2 \mathrm{H}, J=7.6 \mathrm{~Hz}), 7.32$ $(\mathrm{dd}, 2 \mathrm{H}, J=8.7,7.6 \mathrm{~Hz}), 7.23(\mathrm{~d}, 1 \mathrm{H}, J=7.2 \mathrm{~Hz}), 6.1(\mathrm{dt}, 1 \mathrm{H}, J=$ 5.6, $2.2 \mathrm{~Hz}), 5.8(\mathrm{dt}, 1 \mathrm{H}, J=5.5,2.2 \mathrm{~Hz}), 2.63(\mathrm{~m}, 1 \mathrm{H}), 2.45(\mathrm{~m}$, $1 \mathrm{H}), 2.24(\mathrm{t}, 2 \mathrm{H}, J=6.3 \mathrm{~Hz}), 1.99(\mathrm{~s}, 1 \mathrm{H}) ;{ }^{13} \mathrm{C}-\mathrm{NMR}(100 \mathrm{MHz}$, $\left.\mathrm{CDCl}_{3}\right) \delta 146.9,136.5,134.7,128.3,128.1,126.7,126.0,124.7$, $86.9,42.0,31.5$.

\section{6-Methxymethoxy-2-phenylbicyclo[3.2.1]oct-3-en-2-ol (9a)}

IR (neat): $3441 \mathrm{~cm}^{-1} ;{ }^{1} \mathrm{H}-\mathrm{NMR} \delta 7.50(\mathrm{~d}, 2 \mathrm{H}, J=7.8 \mathrm{~Hz}), 7.33$ (t, $2 \mathrm{H}, J=7.8 \mathrm{~Hz}), 7.25(\mathrm{t}, 1 \mathrm{H}, J=7.8 \mathrm{~Hz}), 6.12(\mathrm{dd}, 1 \mathrm{H}, \mathrm{J}=9.8$,<smiles>COC1CC(O)CC(OC)C1c1ccccc1</smiles>
$6.3 \mathrm{~Hz}), 5.64(\mathrm{dd}, 1 \mathrm{H}, J=9.8,2.2 \mathrm{~Hz}), 4.66(\mathrm{~s}, 2 \mathrm{H}), 4.24(\mathrm{dt}, 1 \mathrm{H}$, $J=9.5,5.4 \mathrm{~Hz}), 3.39(\mathrm{~s}, 3 \mathrm{H}), 2.64(\mathrm{dd}, 1 \mathrm{H}, J=9.8,5.6 \mathrm{~Hz}), 2.37$ (t, $1 \mathrm{H}, J=6.3 \mathrm{~Hz}), 2.24(\mathrm{dd}, 1 \mathrm{H}, J=14.5,9.5 \mathrm{~Hz}), 2.20$ (s, 1H), $2.14(\mathrm{dd}, 1 \mathrm{H}, J=14.5,5.6 \mathrm{~Hz}), 1.67(\mathrm{~d}, 1 \mathrm{H}, J=12.2 \mathrm{~Hz}), 1.35(\mathrm{dt}$, $2 \mathrm{H}, J=12.2,4.9 \mathrm{~Hz}) ;{ }^{13} \mathrm{C}-\mathrm{NMR}\left(100 \mathrm{MHz}, \mathrm{CDCl}_{3}\right) \delta 146.7,132.3$, $130.2,127.8,127.1,126.5,96.0,82.4,79.1,55.5,46.1,38.6,32.4$, 
30.4; MS m/z $261\left(\mathrm{M}^{+}\right), 154(100 \%)$; HRMS Calcd. $\mathrm{C}_{16} \mathrm{H}_{20} \mathrm{O}_{3}$ $\left(\mathrm{M}^{+}\right)$: 260.1412. Found: 260.1386.

\section{3-Methyltricyclo[5.2.1.0 $\left.{ }^{2,6}\right]$ deca-4,8-dien-3-ol (10a)}

IR (neat): $3355 \mathrm{~cm}^{-1} ;{ }^{1} \mathrm{H}-\mathrm{NMR} \delta 6.17(\mathrm{dd}, 1 \mathrm{H}, J=5.3,2.9 \mathrm{~Hz})$, $5.9(\mathrm{dd}, 1 \mathrm{H}, J=5.3,3.3 \mathrm{~Hz}), 5.45(\mathrm{dd}, 1 \mathrm{H}, J=7.9,4.3 \mathrm{~Hz}), 3.32$ (dd, 1H, J = 7.9, $4.4 \mathrm{~Hz}$ ), 2.94 (s, 1H), 2.9 (s, 1H), 2.59 (dd, 1H, J $=7.8,4.1 \mathrm{~Hz}), 1.55(\mathrm{t}, 1 \mathrm{H}, J=13.1 \mathrm{~Hz}), 1.42(\mathrm{~d}, 2 \mathrm{H}, J=6.6 \mathrm{~Hz})$, $1.32(\mathrm{~s}, 3 \mathrm{H}) ;{ }^{13} \mathrm{C}-\mathrm{NMR}\left(100 \mathrm{MHz}, \mathrm{CDCl}_{3}\right) \delta 139.4,135.3,133.1$, 132.7, 54.0, 53.5, 52.4, 46.7, 45.1, 30.7; MS m/z $162\left(\mathrm{M}^{+}\right), 66$ (100\%); HRMS Calcd. $\mathrm{C}_{11} \mathrm{H}_{14} \mathrm{O}\left(\mathrm{M}^{+}\right)$: 162.1045. Found: 162.1016.

\section{1-Phenyl-2-cyclohepten-1-ol (11a)}

mp 42-43 ${ }^{\circ} \mathrm{C}$ (recrystallized from hexane); IR (neat): $3254 \mathrm{~cm}^{-1}$; ${ }^{1} \mathrm{H}-\mathrm{NMR} \delta 7.50(\mathrm{dt}, 2 \mathrm{H}, J=7.9,1.0 \mathrm{~Hz}), 7.34(\mathrm{t}, 2 \mathrm{H}, J=7.9 \mathrm{~Hz})$, 7.25 (tt, $1 \mathrm{H}, J=7.9,1.0 \mathrm{~Hz}), 5.96(\mathrm{dt}, 1 \mathrm{H}, J=11.7,5.8 \mathrm{~Hz}), 5.76$ $(\mathrm{d}, 1 \mathrm{H}, J=11.7 \mathrm{~Hz}), 2.32-2.15(\mathrm{~m}, 2 \mathrm{H}), 2.15(\mathrm{~m}, 2 \mathrm{H}), 1.97(\mathrm{~s}, 1 \mathrm{H})$, 1.83-1.70 (m, 1H), 1.70-1.55 (m, 2H), $1.45(\mathrm{~m}, 1 \mathrm{H}) ;{ }^{13} \mathrm{C}-\mathrm{NMR}$ $\left(100 \mathrm{MHz}, \mathrm{CDCl}_{3}\right) \delta 146.7,137.4,131.7,128.1,126.9,125.6$, 78.5, 41.7, 27.6, 26.7, 23.4; MS m/z $188\left(\mathrm{M}^{+}\right), 188$ (100\%); HRMS Calcd. $\mathrm{C}_{13} \mathrm{H}_{16} \mathrm{O}\left(\mathrm{M}^{+}\right)$: 188.1201. Found: 188.1193 .

\section{5-Methyl-2-phenylcyclopent-3-en-1-ol (12a)}

IR (neat): $3389 \mathrm{~cm}^{-1} ;{ }^{1} \mathrm{H}-\mathrm{NMR} \delta 7.43(\mathrm{dd}, 2 \mathrm{H}, J=8.5 \mathrm{~Hz}), 7.30$ (ddt, $2 \mathrm{H}, J=8.5,7.6,1.2 \mathrm{~Hz}), 7.23(\mathrm{~d}, 1 \mathrm{H}, J=7.6 \mathrm{~Hz}), 5.72(\mathrm{~d}$, $1 \mathrm{H}, J=15.9 \mathrm{~Hz}$ ), 2.31 (oct, $1 \mathrm{H}, J=6.6 \mathrm{~Hz}$ ), $1.61(\mathrm{~s}, 3 \mathrm{H}), 1.00$ $(\mathrm{dd}, 6 \mathrm{H}, J=6.6,1.5 \mathrm{~Hz}) ;{ }^{13} \mathrm{C}-\mathrm{NMR}\left(100 \mathrm{MHz}, \mathrm{CDCl}_{3}\right) \delta 147.2$, 135.8, 133.9, 128.0, 126.6, 125.1, 74.3,30.8, 30.3, 22.4. ; MS m/z $190\left(\mathrm{M}^{+}\right), 147$ (100\%); HRMS Calcd. $\mathrm{C}_{13} \mathrm{H}_{18} \mathrm{O}\left(\mathrm{M}^{+}\right)$: 190.1358. Found: 190.1397. 


\section{1,5-Dimethylnon-3-en-5-ol (13a)}

IR (neat): $3390 \mathrm{~cm}^{-1} ;{ }^{1} \mathrm{H}-\mathrm{NMR} \delta 5.56(\mathrm{dd}, 1 \mathrm{H}, J=15.8,6.5 \mathrm{~Hz})$, $5.45(\mathrm{~d}, 1 \mathrm{H}, J=15.8 \mathrm{~Hz}), 2.28(\mathrm{dt}, 1 \mathrm{H}, J=12.3,6.6 \mathrm{~Hz}), 1.821(\mathrm{~s}$, $1 \mathrm{H}), 1.49(\mathrm{~m}, 2 \mathrm{H}), 1.31(\mathrm{~m}, 4 \mathrm{H}), 1.25(\mathrm{~s}, 3 \mathrm{H}), 0.99(\mathrm{~d}, 6 \mathrm{H}, J=6.6$ $\mathrm{Hz}), 0.90(\mathrm{t}, 3 \mathrm{H}, J=6.7 \mathrm{~Hz}) ;{ }^{13} \mathrm{C}-\mathrm{NMR}\left(100 \mathrm{MHz}, \mathrm{CDCl}_{3}\right) \delta 134.7$, 133.8, 72.7, 42.6, 30.7, 27.8, 26.2, 23.1, 22.5, 14.1; MS m/z 169 $\left(\mathrm{M}^{+}-\mathrm{H}\right), 113(100 \%)$; HRMS Calcd. $\mathrm{C}_{11} \mathrm{H}_{21} \mathrm{O}\left(\mathrm{M}^{+}-\mathrm{H}\right): 169.1592$. Found: 169.1573.

\section{Typical experimental procedure for IBX-mediated oxidative rearrangement}

To a stirred solution of tertially allylic alcohol (1a) $(100 \mathrm{mg}, 574 \mu \mathrm{mol})$ in DMSO (3 ml) was added $\mathrm{IBX}^{1}(320 \mathrm{mg}, 1.15 \mathrm{mmol})$ at room temperature. After IBX was dissolved, the reaction was warmed to $55^{\circ} \mathrm{C}$ and stirred for $1 \mathrm{~h}$. After cooling to rt, water was added and the mixture was extracted with diethyl ether. The organic extract was washed with brine, dried $\left(\mathrm{MgSO}_{4}\right)$, and concentrated. The residue was purified by silica gel column chromatography (EtOAc-hexane $=1: 4)$ to give enone $(\mathbf{1 b})(84 \mathrm{mg}, 488 \mu \mathrm{mol}, 85 \%)$ as a colorless crystal and the dehydrated product (12 mg, $77 \mu \mathrm{mol}, 13 \%)$. 


\section{3-Phenyl-2-cyclohexen-1-one (1b)}

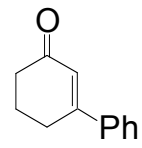

$\mathrm{mp} 64-65{ }^{\circ} \mathrm{C}$ (recrystallized from hexane); IR (neat): $1663 \mathrm{~cm}^{-1}$; ${ }^{1} \mathrm{H}$-NMR $\delta$ 7.56-7.50 (m, 2H), 7.44-7.38 (m, 3H), $6.42(\mathrm{~s}, 1 \mathrm{H})$, $2.78(\mathrm{td}, 2 \mathrm{H}, J=6.1,2.4 \mathrm{~Hz}), 2.49(\mathrm{t}, 2 \mathrm{H}, J=6.1 \mathrm{~Hz}), 2.16$ (qt, $2 \mathrm{H}, J=6.1 \mathrm{~Hz}) ;{ }^{13} \mathrm{C}-\mathrm{NMR}\left(100 \mathrm{MHz}, \mathrm{CDCl}_{3}\right) \delta 199.7,159.6$, 138.7, 129.9, 128.6, 126.0, 125.3, 37.3, 28.1, 22.9; MS m/z 172 $\left(\mathrm{M}^{+}\right), 144$ (100\%); HRMS Calcd. $\mathrm{C}_{15} \mathrm{H}_{16} \mathrm{O}_{3}: 172.0888$. Found: 172.0856 .

\section{3-Butyl-2-cyclohexen-1-one (2b)}

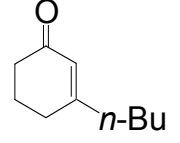

IR (neat): $1670 \mathrm{~cm}^{-1} ;{ }^{1} \mathrm{H}-\mathrm{NMR} \delta 5.87$ (t, $1 \mathrm{H}, J=1.4 \mathrm{~Hz}$ ), 2.35 (ddd, $2 \mathrm{H}, J=7.5,6.7,2.2 \mathrm{~Hz}), 2.29(\mathrm{t}, 2 \mathrm{H}, J=6.1 \mathrm{~Hz}), 2.21(\mathrm{t}$, $2 \mathrm{H}, J=7.5 \mathrm{~Hz}), 2.05-1.95(\mathrm{~m}, 2 \mathrm{H}), 1.53-1.45(\mathrm{~m}, 2 \mathrm{H}), 1.39-1.29$ $(\mathrm{m}, 2 \mathrm{H}), 0.92(\mathrm{td}, 2 \mathrm{H}, J=7.3,2.2 \mathrm{~Hz}) ;{ }^{13} \mathrm{C}-\mathrm{NMR}(100 \mathrm{MHz}$, $\left.\mathrm{CDCl}_{3}\right) \delta 199.7,166.5,125.4,37.7,37.3,29.6,29.0,22.7,22.3$, 13.8; MS m/z $152\left(\mathrm{M}^{+}\right), 82(100 \%)$; HRMS Calcd. $\mathrm{C}_{10} \mathrm{H}_{10} \mathrm{O}$ : 152.1201. Found: 152.1180 .

\section{4-(3-oxo-1-cyclohexenyl)-benzyl acetate (3b)}

IR (neat): $1741,1665 \mathrm{~cm}^{-1} ;{ }^{1} \mathrm{H}-\mathrm{NMR} \delta 7.53(\mathrm{~d}, 2 \mathrm{H}, J=8.3 \mathrm{~Hz}$ ),

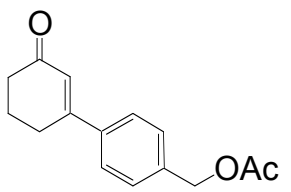
$7.39(\mathrm{~d}, 2 \mathrm{H}, J=8.3 \mathrm{~Hz}), 6.41(\mathrm{~s}, 1 \mathrm{H}), 5.13(\mathrm{~s}, 2 \mathrm{H} \mathrm{Hz}), 2.76(\mathrm{t}, 2 \mathrm{H}$, $J=6.0 \mathrm{~Hz}), 2.49(\mathrm{t}, 2 \mathrm{H}, J=6.0 \mathrm{~Hz}), 2.16(\mathrm{qt}, 2 \mathrm{H}, J=6.0 \mathrm{~Hz})$,

$2.12(\mathrm{~s}, 3 \mathrm{H}) ;{ }^{13} \mathrm{C}-\mathrm{NMR}\left(100 \mathrm{MHz}, \mathrm{CDCl}_{3}\right) \delta 199.5,170.5,159.0$, 138.5, 137.7, 128.3, 126.3, 125.4, 65.6, 37.2, 28.1, 22.8, 21.0; MS $\mathrm{m} / \mathrm{z} 244\left(\mathrm{M}^{+}\right), 156(100 \%)$; HRMS Calcd. $\mathrm{C}_{15} \mathrm{H}_{10} \mathrm{O}_{3}: 244.1099$. Found: 244.1097. 


\section{3-(4-Methoxymethoxymethylphenyl)-2-cyclohexen-1-one (4b)}

$\mathrm{mp}$ 45.5-46.5 ${ }^{\circ} \mathrm{C}$ (recrystallized from hexane-diethyl ether); IR

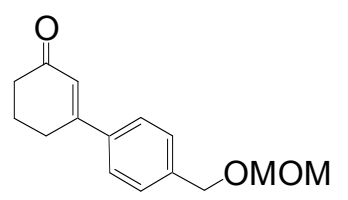
(neat): $1660 \mathrm{~cm}^{-1} ;{ }^{1} \mathrm{H}-\mathrm{NMR} \delta 7.52(\mathrm{~d}, 2 \mathrm{H}, J=8.2 \mathrm{~Hz}), 7.40(\mathrm{~d}$, $2 \mathrm{H}, J=8.2 \mathrm{~Hz}), 6.42(\mathrm{~s}, 1 \mathrm{H}), 4.72(\mathrm{~s}, 2 \mathrm{H}), 4.62(\mathrm{~s}, 2 \mathrm{H}), 3.42(\mathrm{~s}$, $3 \mathrm{H}), 2.77(\mathrm{t}, 2 \mathrm{H}, J=6.0 \mathrm{~Hz}), 2.49(\mathrm{t}, 2 \mathrm{H}, J=6.0 \mathrm{~Hz}), 2.16(\mathrm{qt}, 2 \mathrm{H}$, $J=6.0 \mathrm{~Hz}) ;{ }^{13} \mathrm{C}-\mathrm{NMR}\left(100 \mathrm{MHz}, \mathrm{CDCl}_{3}\right) \delta 199.6,159.2,139.9$, 138.0, 127.9, 126.0, 125.2, 95.7, 68.5, 55.4, 37.2, 28.1, 22.8; MS m/z $246\left(\mathrm{M}^{+}\right), 246(100 \%)$; HRMS Calcd. $\mathrm{C}_{15} \mathrm{H}_{16} \mathrm{O}_{3}: 246.1256$. Found: 246.1261.

\section{3-[4-(tert-Butyldiphenylsilanyloxymethyl)phenyl]-2-cyclohexenen-1-one (5b)}

IR (neat): $1667 \mathrm{~cm}^{-1} ;{ }^{1} \mathrm{H}-\mathrm{NMR} \delta 7.68(\mathrm{dd}, 4 \mathrm{H}, \mathrm{J}=6.7,1.1 \mathrm{~Hz})$,

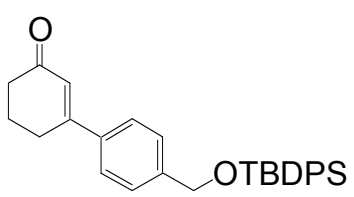
$7.51(\mathrm{~d}, 2 \mathrm{H}, J=8.1 \mathrm{~Hz}), 7.50-7.31(\mathrm{~m}, 6 \mathrm{H}), 7.40(\mathrm{~d}, 2 \mathrm{H}, J=8.1$ $\mathrm{Hz}), 6.43(\mathrm{~s}, 1 \mathrm{H}), 4.79(\mathrm{~s}, 2 \mathrm{H}), 2.78(\mathrm{t}, 2 \mathrm{H}, J=6.0 \mathrm{~Hz}), 2.49(\mathrm{t}$, $2 \mathrm{H}, J=6.0 \mathrm{~Hz}), 2.15(\mathrm{qt}, 2 \mathrm{H}, J=6.0 \mathrm{~Hz}), 1.10(\mathrm{~s}, 9 \mathrm{H}) ;{ }^{13} \mathrm{C}-\mathrm{NMR}$ $\left(100 \mathrm{MHz}, \mathrm{CDCl}_{3}\right) \delta 199.7,159.4,143.1,137.2,135.4,133.2$, 129.7, 127.7, 126.1, 125.9, 124.9, 65.1, 37.3, 28.1, 26.9, 22.9, 19.4; MS m/z $383\left(\mathrm{M}^{+}-t \mathrm{Bu}\right), 383$ (100\%); HRMS Calcd. $\mathrm{C}_{25} \mathrm{H}_{23} \mathrm{O}_{2} \mathrm{Si}$ : 383.1467. Found: 383.1458.

\section{3-[4-(tert-Butyldimethylsiloxymethyl)phenyl]-2-cyclohexen-1-one (6b)}

mp 50-51 ${ }^{\circ} \mathrm{C}$ (recrystallized from hexane); IR (neat): $1660 \mathrm{~cm}^{-1}$;

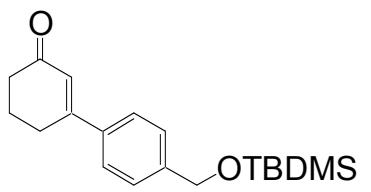
${ }^{1} \mathrm{H}-\mathrm{NMR} \delta 7.51(\mathrm{~d}, 2 \mathrm{H}, J=8.1 \mathrm{~Hz}), 7.36(\mathrm{~d}, 2 \mathrm{H}, J=8.1 \mathrm{~Hz}), 6.42$ $(\mathrm{s}, 1 \mathrm{H}), 4.72(\mathrm{~s}, 2 \mathrm{H}), 4.76(\mathrm{~s}, 2 \mathrm{H}), 2.77(\mathrm{t}, 2 \mathrm{H}, J=5.6 \mathrm{~Hz}), 2.49(\mathrm{t}$, $2 \mathrm{H}, J=6.3 \mathrm{~Hz}), 2.16(\mathrm{tt}, 2 \mathrm{H}, J=6.3,5.6 \mathrm{~Hz}) ;{ }^{13} \mathrm{C}-\mathrm{NMR}$ $\left(100 \mathrm{MHz}, \mathrm{CDCl}_{3}\right) \delta 199.8,159.5,143.6,137.1,126.1,125.9$, 124.9, 64.5, 37.3, 28.1, 26.0, 22.9, 18.5; MS m/z $316\left(\mathrm{M}^{+}\right), 259$ (100\%); HRMS Calcd. $\mathrm{C}_{19} \mathrm{H}_{28} \mathrm{O}_{2} \mathrm{Si}$ : 316.1859 Found: 316.1845 . 


\section{4-(3-Oxo-1-cyclohexenyl)-benzaldehyde (S6)}

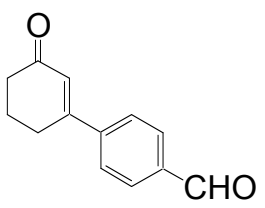

IR (neat): 1700, $1668 \mathrm{~cm}^{-1} ;{ }^{1} \mathrm{H}-\mathrm{NMR} \delta 10.0(\mathrm{~s}, 1 \mathrm{H}), 7.92(\mathrm{~d}, 2 \mathrm{H}$, $J=8.3 \mathrm{~Hz}), 7.68(\mathrm{~d}, 2 \mathrm{H}, J=8.3 \mathrm{~Hz}), 6.46(\mathrm{~s}, 1 \mathrm{H}), 2.80(\mathrm{t}, 2 \mathrm{H}, J=$ $5.6 \mathrm{~Hz}), 2.52(\mathrm{t}, 2 \mathrm{H}, J=6.8 \mathrm{~Hz}), 2.20(\mathrm{tt}, 2 \mathrm{H}, J=6.8,5.6 \mathrm{~Hz}) ;{ }^{13} \mathrm{C}-$ NMR $\left(100 \mathrm{MHz}, \mathrm{CDCl}_{3}\right) \delta 199.2,191.3,157.9,144.6,136.8,29.9$, $127.1,126.6,37.2,28.1,22.8$

\section{3-[3-(tert-Butyldimethylsiloxy)phenyl]-2-cyclohexen-1-one (7b)}

IR (neat): $1668 \mathrm{~cm}^{-1} ;{ }^{1} \mathrm{H}-\mathrm{NMR} \delta 7.26(\mathrm{dd}, 1 \mathrm{H}, J=7.9,7.7 \mathrm{~Hz}), 7.12$ $(\mathrm{dd}, 1 \mathrm{H}, J=7.7,2.2 \mathrm{~Hz}), 6.99(\mathrm{t}, 1 \mathrm{H}, J=2.2 \mathrm{~Hz}), 6.88(\mathrm{dd}, 1 \mathrm{H}, J$

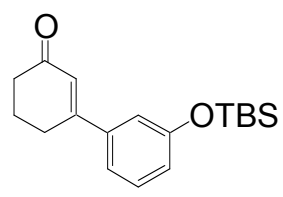
$=7.9,2.2 \mathrm{~Hz}), 6.38(\mathrm{t}, 1 \mathrm{H}, J=1.3 \mathrm{~Hz}), 2.74(\mathrm{td}, 2 \mathrm{H}, J=6.0,1.3$ $\mathrm{Hz}), 2.48(\mathrm{t}, 2 \mathrm{H}, J=6.0 \mathrm{~Hz}), 2.15(\mathrm{qt}, 2 \mathrm{H}, J=6.0 \mathrm{~Hz}), 1.00(\mathrm{~s}$, 9H), $0.21(\mathrm{~s}, 6 \mathrm{H}) ;{ }^{13} \mathrm{C}-\mathrm{NMR}\left(100 \mathrm{MHz}, \mathrm{CDCl}_{3}\right) \delta$ 199.7, 159.4, 155.8, 140.1, 129.6, 125.3, 121.5, 119.0, 117.7, 37.3, 28.1, 25.7, 22.8, 18.2; MS m/z $302\left(\mathrm{M}^{+}\right), 245$ (100\%); HRMS Calcd. $\mathrm{C}_{18} \mathrm{H}_{26} \mathrm{O}_{2} \mathrm{Si}: 302.1702$. Found: 302.1692 .

\section{3-Phenyl-2-cyclopenten-1-one (8b)}

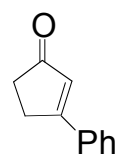

mp 81-82 ${ }^{\circ} \mathrm{C}$ (recrystallized from hexane); IR (neat): $1682 \mathrm{~cm}^{-1}$; ${ }^{1} \mathrm{H}-\mathrm{NMR} \delta$ 7.58-7.60 (m, 2H), 7.47-7.38 (m, 3H), $6.56(\mathrm{~d}, 1 \mathrm{H}, J=$ $1.0 \mathrm{~Hz}), 3.04-2.98(\mathrm{~m}, 2 \mathrm{H}), 2.60-2.52(\mathrm{~m}, 2 \mathrm{H}) ;{ }^{13} \mathrm{C}-\mathrm{NMR}$ $\left(100 \mathrm{MHz}, \mathrm{CDCl}_{3}\right) \delta 209.0,174.0,133.9,131.0,128.7,127.3$, 126.6, 35.2, 28.6; MS m/z $158\left(\mathrm{M}^{+}\right), 158$ (100\%); HRMS Calcd. $\mathrm{C}_{11} \mathrm{H}_{12} \mathrm{O}: 158.0732$. Found: 158.0723 .

\section{7-Methxymethoxy-4-phenylbicyclo[3.2.1]oct-3-en-2-one (9b)}

IR (neat): $1668 \mathrm{~cm}^{-1} ;{ }^{1} \mathrm{H}-\mathrm{NMR} \delta 7.55(\mathrm{~d}, 2 \mathrm{H}, J=6.7 \mathrm{~Hz})$, 7.43-7.39 (m, 3H), 6.29 (s, 1H), 4.75(ddd, $1 \mathrm{H}, J=9.3,6.3,3,3$ $\mathrm{Hz}), 4.69(\mathrm{~d}, 1 \mathrm{H}, J=6.7 \mathrm{~Hz}), 4.53(\mathrm{~d}, 1 \mathrm{H}, J=6.7 \mathrm{~Hz}) 3.38-3.26$ (m, 2H), $3.33(\mathrm{~s}, 3 \mathrm{H}), 2.59(\mathrm{ddd}, 1 \mathrm{H}, J=13.8,8.5,5.4 \mathrm{~Hz}), 2.22$ (dd, $1 \mathrm{H}, \mathrm{J}=11.8,2.5 \mathrm{~Hz}), 1.89(\mathrm{ddd}, 1 \mathrm{H}, \mathrm{J}=11.8,4.4,4,2 \mathrm{~Hz})$, $1.82(\mathrm{dt}, 2 \mathrm{H}, J=13.8,3.1 \mathrm{~Hz}) ;{ }^{13} \mathrm{C}-\mathrm{NMR}\left(100 \mathrm{MHz}, \mathrm{CDCl}_{3}\right) \delta$ 
199.8, 167.1, 137.2, 129.9, 128.7, 126.2, 123.9, 95.7, 76.6, 55.6, 54.5, 39.3, 38.7, 38.2; MS m/z $258\left(\mathrm{M}^{+}\right), 198$ (100\%); HRMS Calcd. $\mathrm{C}_{16} \mathrm{H}_{18} \mathrm{O}_{3}$ : 258.1256. Found: 258.1266.

\section{5-Methyltricyclo[5.2.1.0 $\left.{ }^{2,6}\right]$ deca-4,8-dien-3-one (10b)}

IR (neat): $1695 \mathrm{~cm}^{-1} ;{ }^{1} \mathrm{H}-\mathrm{NMR} \delta 5.98(\mathrm{dd}, 1 \mathrm{H}, J=5.6,3.0 \mathrm{~Hz})$, $5.79(\mathrm{dd}, 1 \mathrm{H}, J=5.6,2.9 \mathrm{~Hz}), 5.69(\mathrm{~s}, 1 \mathrm{H}), 3.22(\mathrm{t}, 1 \mathrm{H}, J=4.9$

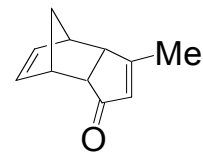
$\mathrm{Hz}), 3.18(\mathrm{~s}, 1 \mathrm{H}), 3.01(\mathrm{~s}, 1 \mathrm{H}), 2.85(\mathrm{t}, 1 \mathrm{H}, J=5.1 \mathrm{~Hz}), 1.98(\mathrm{~s}$, $3 \mathrm{H}), 1.77(\mathrm{~d}, 1 \mathrm{H}, J=8.3 \mathrm{~Hz}), 1.59(\mathrm{~d}, 1 \mathrm{H}, \mathrm{J}=8.7 \mathrm{~Hz}) ;{ }^{13} \mathrm{C}-\mathrm{NMR}$ $\left(100 \mathrm{MHz}, \mathrm{CDCl}_{3}\right) \delta 209.8,178.06,133.4,132.9,131.4,52.4$, 51.7, 51.1, 44.3, 43.3, 18.2; MS m/z $160\left(\mathrm{M}^{+}\right), 66$ (100\%); HRMS Calcd. $\mathrm{C}_{11} \mathrm{H}_{12} \mathrm{O}$ : 160.0888 . Found: 160.0877 .

\section{3-Phenyl-2-cyclohepten-1-one (11b)}

IR (neat): $1651 \mathrm{~cm}^{-1} ;{ }^{1} \mathrm{H}-\mathrm{NMR} \delta 7.44(\mathrm{~d}, 2 \mathrm{H}, J=8.8 \mathrm{~Hz}), 7.41-$

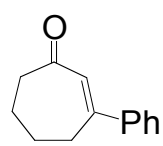
$7.31(\mathrm{~m}, 3 \mathrm{H}), 6.30(\mathrm{~s}, 1 \mathrm{H}), 2.88(\mathrm{t}, 2 \mathrm{H}, J=5.6 \mathrm{~Hz}), 2.68(\mathrm{t}, 2 \mathrm{H}, J$ $=5.8 \mathrm{~Hz}), 1.95(\mathrm{dt}, 2 \mathrm{H}, \mathrm{J}=6.3,5.8 \mathrm{~Hz}), 1.91(\mathrm{dt}, 2 \mathrm{H}, J=6.3,5.6$ $\mathrm{Hz}) ;{ }^{13} \mathrm{C}-\mathrm{NMR}\left(100 \mathrm{MHz}, \mathrm{CDCl}_{3}\right) \delta 204.1,157.4,142.3,130.3$, 128.8, 128.4, 126.3, 42.0, 32.0, 25.3, 21.0; MS m/z $186\left(\mathrm{M}^{+}\right), 157$ (100\%); HRMS Calcd. $\mathrm{C}_{13} \mathrm{H}_{14} \mathrm{O}: 186.1045$. Found: 186.1020 .

\section{References}

1. Frigerio, M.; Santagostino, M.; Sputore. S. J. Org. Chem. 1999, 64, 4537.

2. Ireland, R. E.; Liu, L. J. Org. Chem. 1993, 58, 2899.

3. Kazmierczak, P.; Skulski, L.; Kraszkiewicz, L. Molecules 2001, 6, 881.

4. Saltzman, H.; Sharefkin, J. G. Org. Syn. Coll. Vol.5, 658.

5. The bycyclic and tricyclic enones destined to 9a and 10a were prepared according to the literature procedures. For 9a, see: Nagata, H.; Miyazawa, N.; Ogasawara, K. Org. Lett. 2001, 4, 1737. For 10a, see: Takano, S.; Inomata, K.; Ogasawara, K. J. Chem. Soc., Chem. 
Commun. 1989, 271

6. Schlosser, M. Organometallics in Synthesis; Schlosser, M. Ed.; Wiley: Chichester, 1994, pp $1-166$. 